\title{
Interventions to Minimize Jet Lag After Westward and Eastward Flight
}

\author{
Gregory D. Roach* and Charli Sargent \\ Appleton Institute for Behavioural Science, Central Queensland University, Adelaide, SA, Australia
}

Keywords: time zone, adaptation, sleep, circadian, light, melatonin, exercise, athlete

\section{INTRODUCTION}

Air travel across several time zones, i.e., transmeridian flight, causes negative effects-some of which occur during flight and some of which occur in the days after flight. Anecdotally, these effects are often referred to collectively as jet lag, but they are actually two separate phenomena-travel fatigue and jet lag_-each with their own causes and consequences (Waterhouse et al., 2004). Travel fatigue refers to a collection of symptoms that occur during or immediately after long flights. These symptoms include fatigue, disorientation, and headache (Waterhouse et al., 2004) - primarily caused by the sleep loss, dehydration, hypoxia, and discomfort associated with being in an aircraft with confined space, recline-restricted seats, low air pressure, low humidity, etc., for 8-14 h (Brown et al., 2001; Roach et al., 2018). In contrast, jet lag refers to a collection of symptoms that occur in the days after flight across three or more time zones. These symptoms include headache, irritability,

\section{OPEN ACCESS}

Edited by:

Till Roenneberg,

Ludwig Maximilian University of

Munich, Germany

Reviewed by:

Hanspeter Herzel,

Humboldt University of

Berlin, Germany

Sara Montagnese,

University of Padova, Italy

*Correspondence:

Gregory D. Roach

greg.roach@cqu.edu.au

Specialty section:

This article was submitted to

Chronobiology,

a section of the journal

Frontiers in Physiology

Received: 09 April 2019

Accepted: 09 July 2019

Published: 31 July 2019

Citation:

Roach GD and Sargent C (2019) Interventions to Minimize Jet Lag After Westward and Eastward Flight.

Front. Physiol. 10:927.

doi: 10.3389/fphys.2019.00927 daytime sleepiness, difficulty sleeping at night, poor mental and physical performance, and poor gastrointestinal function (Waterhouse et al., 2004) - primarily caused by the mismatch between the circadian system, or internal body clock, which is synchronized to time cues in the departure time zone, and the desired timing of sleep and wake, which are typically synchronized to time cues in the destination time zone.

In August 2020, the Olympic Games will be held in Tokyo, Japan. Athletes will travel from all over the world to compete in the Games, and many will have to travel across several time zones. For example, athletes traveling to Japan from North America and Western Europe will face time zone changes of $8-11 \mathrm{~h}$ west and 6-8h east, respectively. Some athletes will travel to Japan, or nearby countries, weeks before their events, while others will arrive in Japan in the days prior to competition. In either case, athletes will want to adjust to the new time zone as quickly as possible so that they can prepare well and/or compete at the highest level.

The purpose of this manuscript is to discuss the causes and consequences of jet lag and to provide examples of how to use judiciously timed light exposure/avoidance and/or exogenous melatonin ingestion to adapt the circadian system to a new time zone after transmeridian flight. These guides could be applied by athletes competing in the Tokyo 2020 Olympic Games, but they could also be applied by athletes traveling to other countries for training or competition, or by non-athletes traveling for business or pleasure.

\section{JET LAG IS CAUSED BY THE DESYNCHRONY BETWEEN THE CIRCADIAN SYSTEM AND LOCAL TIME CUES}

The term "circadian rhythms" refers to rhythms with a period of approximately $24 \mathrm{~h}$ (Halberg, 1959). Many physiological and psychological variables in humans have been shown to alter rhythmically with a $\sim 24 \mathrm{~h}$ period, including core body temperature (Dijk et al., 1992; Zhou et al., 2011a, 2017); cortisol (Scheer et al., 2009), blood pressure (Scheer et al., 2010), heart rate (Scheer et al., 2010), hunger (Sargent et al., 2016), cognitive performance (Dijk et al., 1992; Darwent et al., 2010; Matthews et al., 2010), strength (Reilly et al., 1997; Sargent et al., 2010), balance 
(Sargent et al., 2012b), flexibility (Reilly et al., 1997), dexterity (Matthews et al., 2012a,b), subjective alertness (Dijk et al., 1992; Zhou et al., 2012; Kosmadopoulos et al., 2014), subjective fatigue (Ferguson et al., 2012), subjective sleepiness (Kosmadopoulos et al., 2017), and objective sleepiness (Lavie, 1986; Dijk and Czeisler, 1995; Paech et al., 2010, 2012; Sargent et al., 2012a).

In humans, circadian rhythms in physiological and psychological variables are endogenously generated by a central circadian pacemaker-located within the suprachiasmatic nucleus of the hypothalamus - with a period of approximately $24.2 \mathrm{~h}$ (Czeisler et al., 1999; Zhou et al., 2011b). These rhythms are entrained to the period of a $24 \mathrm{~h}$ day by environmental signals or "zeitgebers", meaning time-givers (Aschoff et al., 1971). Sunlight is the most powerful zeitgeber for humans (Wever et al., 1983; Czeisler et al., 1986), but non-photic stimuli such as social contact, eating, and physical activity, may also play a role (Mistlberger and Skene, 2004). Most peripheral cell types, including those in the major organ systems-heart, lungs, liver, pancreas-also contain their own circadian oscillators, which are kept in coherent phase relationships by the suprachiasmatic nucleus (Yamada and Forger, 2010; Bass, 2012; Schibler et al., 2015).

The circadian system cannot immediately entrain to the timing of zeitgebers in a new time zone (Wever, 1980), so after long-haul flights to the west or east, the circadian system is initially aligned with the timing of zeitgebers at the point of departure rather than zeitgebers at the new location (Winget et al., 1984). A period of desynchrony follows while the circadian system is entrained to the timing of zeitgebers in the new time zone-and it is this period of desynchrony that gives rise to the symptoms of jet lag.

\section{THE TIMING OF THE HUMAN CIRCADIAN SYSTEM CAN BE RESET BY LIGHT, MELATONIN, AND EXERCISE}

The timing of the human sleep/wake and circadian systems are related such that the production of endogenous melatonin begins $\sim 2 \mathrm{~h}$ before habitual bedtime (Burgess et al., 2003b; Burgess and Eastman, 2005), the daily minimum of the core body temperature rhythm (CBTmin), which coincides with the daily low-point of the circadian cycle, occurs $\sim 7 \mathrm{~h}$ after melatonin onset (Cagnacci et al., 1996; Brown et al., 1997; Eastman et al., 2000), and the daily peak of the core body temperature rhythm (CBTmax), which coincides with the daily high-point of the circadian cycle, occurs $\sim 12$ after CBTmin (Dijk et al., 1992). Therefore, a person who normally sleeps from 23:00 to 07:00 will have melatonin onset at $\sim 21: 00$, CBTmin at $\sim 04: 00$, and CBTmax at $\sim 16: 00$ (Figure 1). Maximal sleepiness, and poorest mental/physical performance, occur in the $2-3 \mathrm{~h}$ either side of CBTmin, and maximal alertness, and greatest mental/physical performance, occur in the 2-3 h either side of CBTmax (Dijk et al., 1992). The protocols required to directly assess the timing of melatonin onset and CBTmin are invasive, time-consuming, and costly, so these variables are typically estimated based on the habitual timing of sleep and wake. However, work is currently being conducted to develop biomarkers of circadian phase based on the analysis of white blood cells from a single sample (Ueda et al., 2004; Wittenbrink et al., 2018).

Immediately after westward flight, the circadian system will be running ahead of the local time zone. For example, after a flight from London to Los Angeles ( $8 \mathrm{~h}$ west), when the body clock is ready for bed at 23:00 London time, it will only be 15:00 in Los Angeles. To adjust to the new time zone, the circadian system has to delay, or shift backward, or move later. Conversely, immediately after eastward flight, the circadian system will be running behind the local time zone. For example, after a flight from Los Angeles to London ( $8 \mathrm{~h}$ east), when it is time to get up at 07:00 in London, it is only 23:00 in Los Angeles, so the body clock will be ready for bed. To adjust to the new time zone, the circadian system has to advance, or shift forward, or move earlier.

Delays and advances in the timing of the circadian system can be facilitated by appropriately timed light exposure, melatonin ingestion, and/or exercise (Figure 1). The direction and size of the shift in the timing of the circadian system in response to these stimuli depend on the time of day, or more correctly, the circadian phase, that the stimuli occur. These effects are described by phase response curves (PRCs):

(A) Phase response curve to light. Light exposure in the $\sim 12 \mathrm{~h}$ prior to CBTmin shifts the circadian system backward/later, or causes a phase delay; light exposure in the $\sim 12 \mathrm{~h}$ after CBTmin shifts the circadian system forward/earlier, or causes a phase advance; and the largest shifts occur when light exposure occurs in the 3-6h either side of CBTmin (Czeisler et al., 1989; Khalsa et al., 2003). Furthermore, the degree to which the timing of the circadian system can be shifted by exposure to light depends the duration of the exposure (Rimmer et al., 2000), the intensity of the light (Boivin et al., 1996), and the wavelength of the light (Wright and Lack, 2001; Rüger et al., 2013). The largest phase shifts occur when the duration of exposure is longer, when the intensity of light is higher, and when the wavelength of light is shorter (i.e., blue).

(B) Phase response curves to melatonin. Melatonin ingested in the late afternoon or early evening, a few hours prior to the onset of endogenous melatonin production and several hours prior to CBTmin, shifts the circadian system forward/earlier, or causes a phase advance (Burgess et al., 2008, 2010). Conversely, melatonin ingested in the morning, a few hours after habitual get-up time and several hours after CBTmin, shifts the circadian system backward/later, or causes a phase delay (Burgess et al., 2008, 2010). Separate PRCs have been created for exogenous melatonin at a "physiological" dose of $0.5 \mathrm{mg}$ and at a "pharmacological" dose of $3.0 \mathrm{mg}$. For a $0.5 \mathrm{mg}$ dose, it is estimated that maximum advances occur for ingestion $\sim 10.5 \mathrm{~h}$ before CBTmin, or $\sim 5.5 \mathrm{~h}$ before habitual bedtime, and maximum delays occur for ingestion $\sim 6.5 \mathrm{~h}$ after CBTmin, or $\sim 3.5 \mathrm{~h}$ after habitual get-up time (Burgess et al., 2010). For a $3.0 \mathrm{mg}$ dose, it is estimated that maximum advances occur for ingestion $\sim 11.5 \mathrm{~h}$ before CBTmin, or $\sim 6.5 \mathrm{~h}$ before habitual bedtime, and maximum delays occur for ingestion $\sim 4 \mathrm{~h}$ 


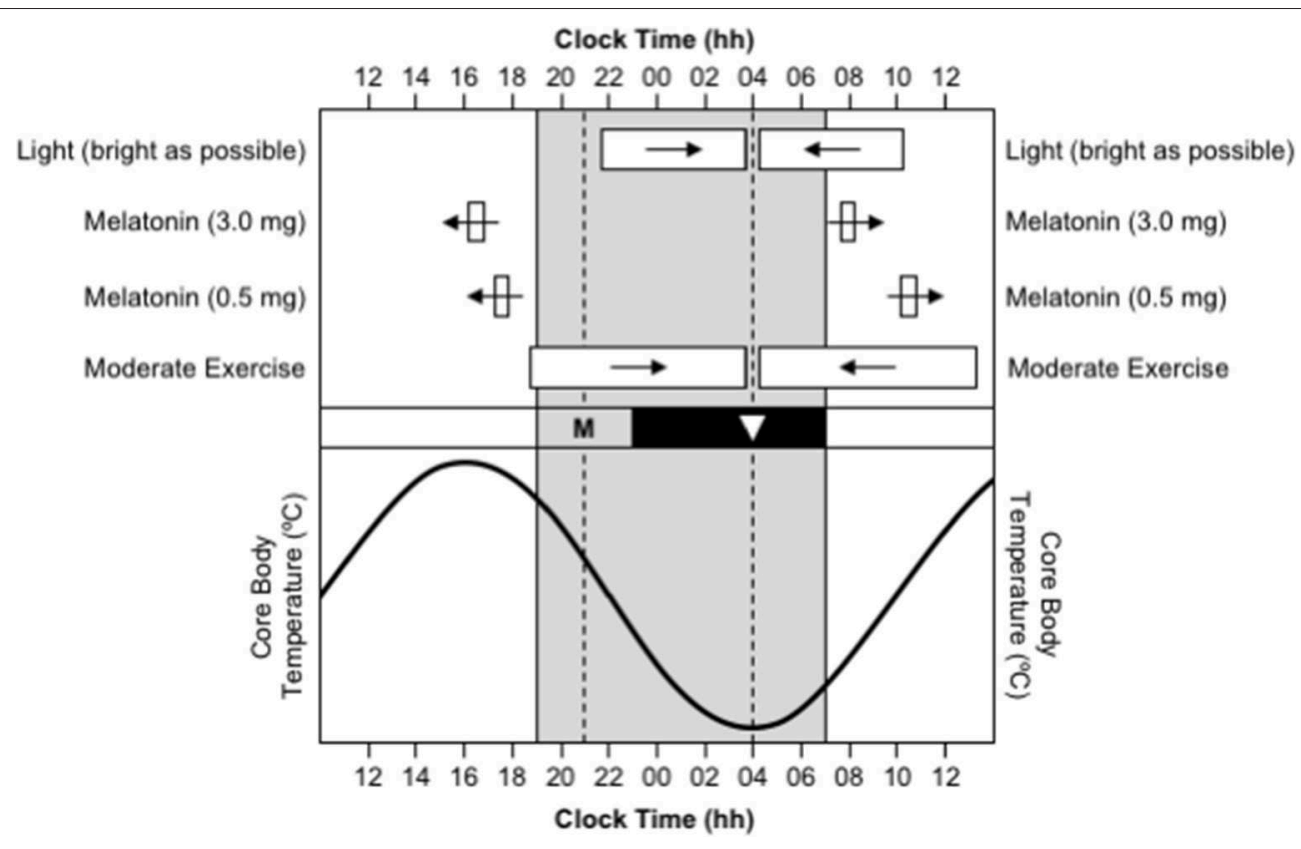

FIGURE 1 | Use of appropriately timed stimuli to shift the timing of the human circadian system. In all sections, the gray background represents night-time. In the bottom section, the sinusoid-shaped line indicates the daily rhythm of core body temperature. In the middle section, the black bar represents habitual bedtime, "M" represents the evening onset of endogenous melatonin production, and the inverted triangle represents the daily minimum of core body temperature. In the top section, the white bars indicate the optimal timing of light exposure, exogenous melatonin ingestion, and moderate-intensity exercise, required to facilitate delays (right-pointing arrows) and advances (left-pointing arrows) in the circadian system. This figure was inspired by a similar figure presented by Waterhouse et al. (2007).

after CBTmin, or $\sim 1 \mathrm{~h}$ after habitual get-up time (Burgess et al., 2008). The two doses of exogenous melatonin produce phase shifts of a similar size, but the $3.0 \mathrm{mg}$ dose produces more reliable phase shifts than the $0.5 \mathrm{mg}$ dose (Burgess et al., 2010). Goodness of fit data were not provided for either PRC, so the apparent precision of the estimated timing of melatonin ingestion for maximum advances and delays should be read with caution.

(C) Phase response curve to exercise. There is some evidence that exercise has phase-shifting properties (Eastman et al., 1995; Buxton et al., 2003), and a phase response curve to a $1 \mathrm{~h}$ bout of moderate-intensity exercise has been published recently (Youngstedt et al., 2019). The PRC indicates that exercise in the $\sim 9 \mathrm{~h}$ prior to CBTmin shifts the circadian system backward/later, or causes a phase delay, and exercise in the $\sim 9 \mathrm{~h}$ after CBTmin shifts the circadian system forward/earlier, or causes a phase advance. However, the protocol to obtain the data to construct this PRC was conducted with moderate-intensity light at 50 lux, instead of low-intensity light at $<10-15$ lux, so a PRC for the effects of exercise independent of light is still to be established.

This manuscript provides examples of how to use light and/or melatonin to shift the timing of the circadian system so that jet lag can be overcome as quickly as possible (Figures 4, 5). Light and melatonin can be used independently, but their phase-shifting effects are additive-particularly for phase advances-so using them together should produce a greater effect than either one on its own (Wirz-Justice et al., 2004; Revell et al., 2006; Burke et al., 2013). In contrast, there is no evidence that the effects of light and exercise are additive, so exercise is not included in the adaptation guides. However, the appropriate times to conduct exercise for its phase-shifting properties coincide with the appropriate times for light exposure.

\section{THE EXPERIENCE OF JET LAG DEPENDS ON THE DIRECTION OF TRAVEL}

The most obvious consequences of jet lag are poor night-time sleep, excessive daytime sleepiness, and poor mental and physical performance (Waterhouse et al., 2004). However, the experience of jet lag greatly depends on the direction of travel. Consider the difference in the manifestation of jet lag between westward and eastward flights over 8 time zones-as occurs with travel between Western Europe $(\mathrm{UTC}+0 \mathrm{~h})$ and the USA's west coast (UTC$8 \mathrm{~h}$ ). Immediately after flying $8 \mathrm{~h}$ west, say from London to Los Angeles, the circadian system is still entrained to the timing of zeitgebers in London, so the daily low-point of the circadian cycle occurs at 04:00 London time, which is 20:00 in Los Angeles, and the daily high-point of the circadian cycle occurs at 16:00 London time, which is 08:00 in Los Angeles (Figure 2A). Consequently, at least initially in Los Angeles, a person will feel sleepy in the evening and they will have difficulty staying asleep until their normal wake time in the morning. If they are an athlete, they will also have difficulty training or competing at their highest 
A Desynchrony after an 8-h time zone shift to the west [e.g., London to Los Angeles]

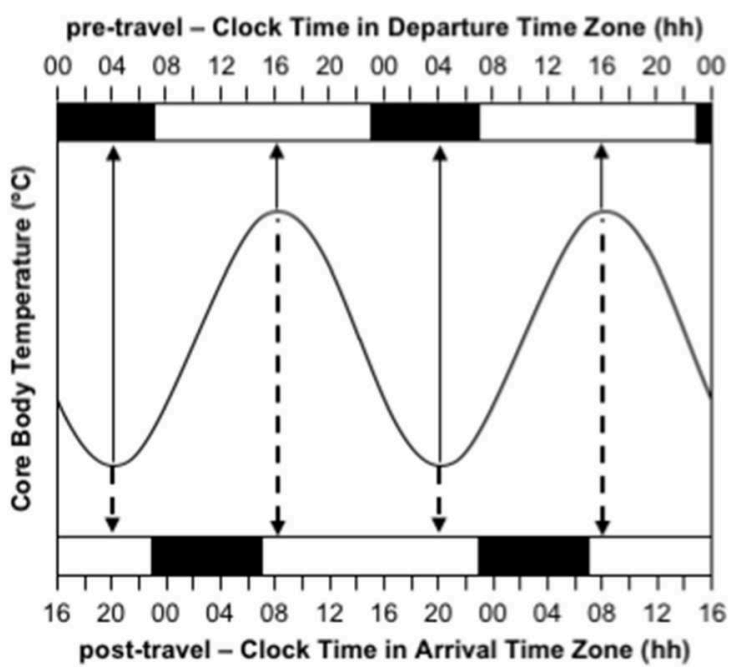

B Desynchrony after an 8-h time zone shift to the east [e.g., Los Angeles to London]

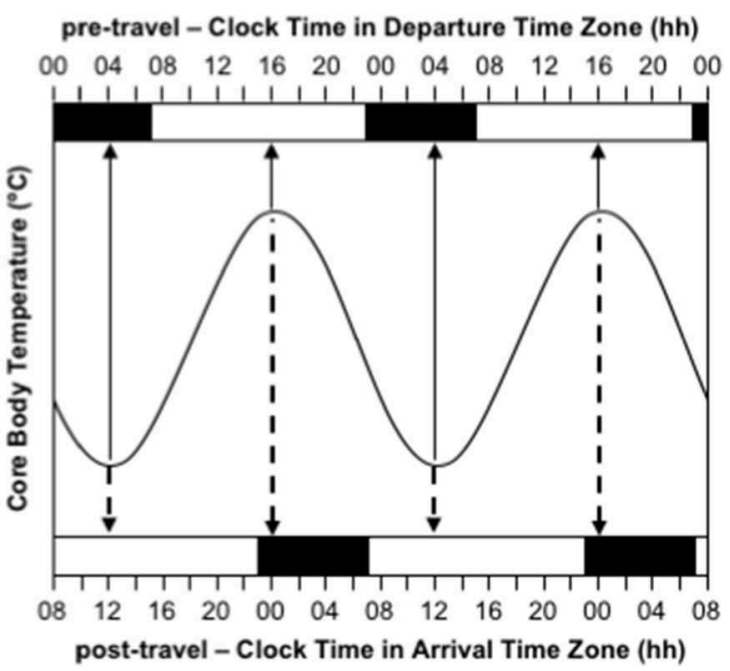

FIGURE 2 | Desynchrony between the circadian system and the desired timing of sleep/wake immediately after time zone shifts to the west (A) and east (B). In both panels, the sinusoid-shaped line represents core body temperature; the solid arrows indicate the timing of the daily minimum and maximum of core body temperature in the departure time zone; the dashed arrows indicate the timing of the daily minimum and maximum of core body temperature in the arrival time zone; the black bars at the top axis represent night-time sleep in the departure time zone; the black bars at the bottom axis represent night-time sleep in the arrival time zone; time is double-potted with $2 \times 24$ h periods. (A) Desynchrony after 8-h time zone shift to the west (e.g., London to Los Angeles). (B) Desynchrony after an 8-h time zone shift to the east (e.g., Los Angeles to London).

level in the evening. In contrast, immediately after flying $8 \mathrm{~h}$ east, say from Los Angeles to London, the circadian system is still entrained to the timing of zeitgebers in Los Angeles, so the daily low-point of the circadian cycle occurs at 04:00 Los Angeles time, which is midday in London, and the daily high-point of the circadian cycle occurs at 16:00 Los Angeles time, which is midnight in London (Figure 2B). Consequently, at least initially in London, a person will feel sleepy in the late morning and early afternoon and they will have difficulty falling asleep at their normal bedtime in the evening. If they are an athlete, they will also have difficulty training and/or competing at their highest level in the late morning and early afternoon.

\section{SUNLIGHT CAN EITHER HELP OR HINDER ADAPTATION TO A NEW TIME ZONE}

From anecdotal reports, it seems that a common perception among laypersons, is that maximizing exposure to sunlight in a new time zone is an effective strategy for overcoming jet lag. In general, this approach will work quite well after westward travel, but it may actually be counterproductive after eastward travel. To overcome jet lag, the circadian system must adjust so that it becomes aligned with the desired timing of sleep and wake in the new time zone. The most effective way to adjust the circadian system, or shift the timing of the body clock, is with exposure to light. However, light exposure, per se, does not cause the circadian system to align with the new time zone. Rather, light exposure shifts the timing of the body clock either backward (delay) or forward (advance), as required after westward and eastward travel, respectively.

Consider the difference in the effectiveness of indiscriminately maximizing exposure to sunlight after westward and eastward travel over 8 time zones. Immediately after flying $8 \mathrm{~h}$ west, say from London to Los Angeles, the circadian system is still entrained to the timing of zeitgebers in London, so CBTmin occurs at 04:00 London time, which is 20:00 in Los Angeles. To adapt to the new time zone in Los Angeles, the circadian system must delay by $8 \mathrm{~h}$, so that CBTmin occurs at the normal time of 04:00 instead of 20:00 (Figure 3A). If a person indiscriminately seeks sunlight during the daytime, they will be exposed to sunlight before CBTmin at 20:00, which will provide a delay signal, and they will be exposed to little or no sunlight after CBTmin, because the sun sets earlier than 20:00 in Los Angeles at most times of year, so they will not receive an advance signal (Figure 3A). In this case, maximizing exposure to sunlight would aid the desired phase delay. In contrast, immediately after flying $8 \mathrm{~h}$ east, say from Los Angeles to London, the circadian system is still entrained to the timing of zeitgebers in Los Angeles, so CBTmin occurs at 04:00 Los Angeles time, which is midday in London. To adapt to the new time zone in London, the circadian system must advance by $8 \mathrm{~h}$, so that CBTmin occurs at the normal time of 04:00 instead of midday (Figure 3B). If a person indiscriminately seeks out sunlight during the daytime, they will be exposed to sunlight before CBTmin at midday, which will provide a delay signal, and they will be exposed to sunlight after CBTmin at midday, which will provide an advance signal (Figure 3B). In this case, maximizing exposure to sunlight would 
A Re-entrainment after an 8-h time zone shift to the west [e.g., London to Los Angeles]

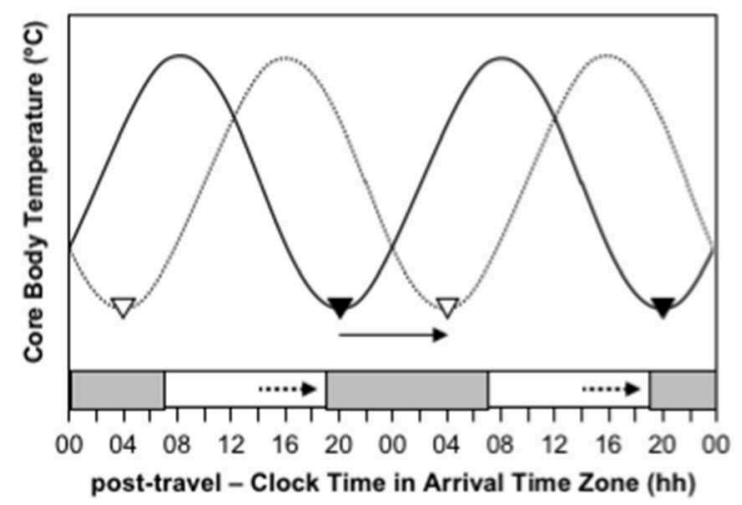

B Re-entrainment after an 8-h time zone shift to the east [e.g., Los Angeles to London]

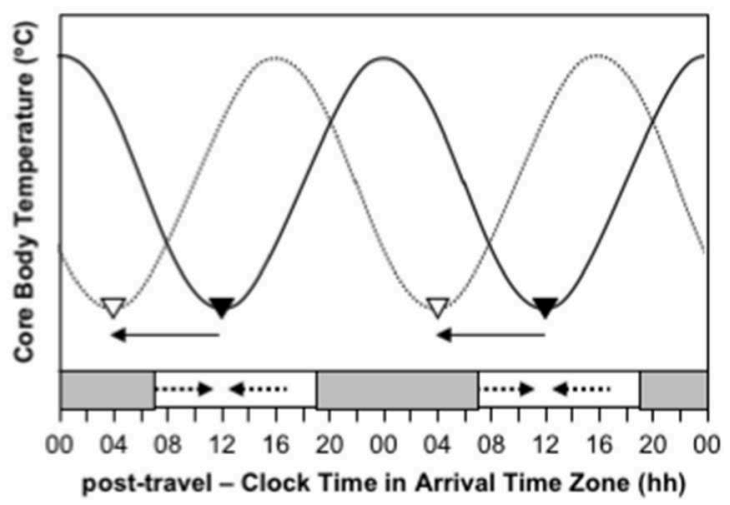

FIGURE 3 | Re-entrainment of the circadian system to local time cues after time zone shifts to the west (A) and east (B). In both panels, the solid sinusoid-shaped line with black inverted triangles represent core body temperature and the timing of its daily minimum immediately after flight; the dashed sinusoid-shaped line with white inverted triangles represent represents core body temperature and the timing of its daily minimum after re-entrainment to the arrival time zone; the gray bars at the bottom axis represent night-time in the arrival time zone; the solid arrows represent the direction of the phase shift required for adaptation to the new time zone; the dashed arrows represent the direction of the phase shift signal provided by light exposure at that time of day; time is double-potted with $2 \times 24 \mathrm{~h}$ periods. (A) Re-entrainment after an 8-h time zone shift to the west (e.g., London to Los Angeles). (B) Re-entrainment after an 8-h time zone shift to the east (e.g., Los Angeles to London).

provide contradictory signals, which would inhibit adaptation to the new time zone. A better approach would be to avoid sunlight before midday and maximize exposure to sunlight in the 3-6h after midday - that would limit the delay signal and maximize the desired advance signal.

\section{ENDOGENOUS MELATONIN HAS HYPNOTIC AND CHRONOBIOTIC PROPERTIES}

Exogenous melatonin is both a chronobiotic and an hypnotoic, i.e., it can shift the timing of the circadian system (Arendt and Skene, 2005), but it can also make it easier to fall asleep and/or stay asleep (van den Heuvel et al., 2005; Zhdanova, 2005). The hypnotic effects of melatonin depend on the time of day, or more correctly, the circadian phase, that the melatonin is ingested (Wyatt et al., 2006). For exogenous melatonin, doses of $\leq 0.5 \mathrm{mg}$ and $1-5 \mathrm{mg}$ are typically considered to be physiological and pharmacological, respectively. If ingested when endogenous melatonin is high, i.e., during body clock nighttime, neither a physiological $0.3 \mathrm{mg}$ dose, nor a pharmacological $5.0 \mathrm{mg}$ dose, of exogenous melatonin increase sleepiness. In contrast, if ingested when endogenous melatonin is low, i.e., during body clock daytime, both doses of exogenous melatonin increase sleepiness to a similar extent. Therefore, if melatonin is used as a chronobiotic at either physiological or pharmacological doses, its potential hypnotic effects should also be considered. This manuscript provides advice on how to exploit the chronobiotic effects of a pharmacological $3.0 \mathrm{mg}$ dose, rather than a physiological $0.5 \mathrm{mg}$ dose, because the higher dose produces more reliable phase shifts than the lower dose (Burgess et al., 2010).

\section{SCHEDULES FOR SHIFTING THE TIMING OF THE CIRCADIAN SYSTEM AFTER TRANSMERIDIAN AIR TRAVEL}

After long-haul flights across multiple time zones, the circadian system is initially aligned with the timing of zeitgebers at the point of departure rather than zeitgebers at the new location. To overcome jet lag, the timing of the circadian system must shift so that it becomes aligned with the new time zone. Adaptation guides have previously been presented for relatively large time zone shifts of 7-9h (Eastman and Burgess, 2009; Revell and Eastman, 2012). The following sub-sections provide examples of how to use light and/or melatonin to shift the timing of the circadian system so that jet lag can be overcome as quickly as possible after rapid time zone changes of $3,6,9$, and $12 \mathrm{~h}$ to the west and east.

The adaptation guides are based on three major assumptions:

- Assumption 1-A person who normally sleeps from 23:00 to 07:00 will have the daily minimum of their core body temperature rhythm (CBTmin) at $\sim 04: 00$. For people with earlier or later bedtimes, the timing of light and melatonin should be adjusted accordingly. For example, a person who normally sleeps from 22:00 to 06:00 will have CBTmin at $\sim 03: 00$ instead of $\sim 04: 00$, so the timing of light and melatonin should be $1 \mathrm{~h}$ earlier than in the examples, and a person who normally sleeps from midnight to 08:00 will have CBTmin at $\sim 05: 00$ instead of $\sim 04: 00$, so the timing of light and melatonin should be $1 \mathrm{~h}$ later than in the examples.

- Assumption 2-A person wants their main daily sleep period to occur at the same local time in the arrival time zone as in their normal time zone (i.e., 23:00 to 07:00 in the 
examples provided). In some cases, this may be difficult to achieve in practice, particularly on the first few days after travel. For example, after a shift of 9 time zones west (Figure 4C), a person may be sleepy in the evening, so they may wish to go to bed earlier than usual (e.g., 21:00-05:00 instead of 23:00-07:00). Similarly, after a shift of 9 time zones east (Figure 5C), a person may not be sleepy in the evening, so they may wish to go to bed later than usual (e.g., 01:00-09:00 instead of 23:00-07:00). In situations where the timing of the main sleep period differs from the timing in the relevant guide, this should not interfere with adaptation provided that light exposure/avoidance and/or melatonin ingestion still occur at the appropriate time.

- Assumption 3-Arrival in the new time zone occurs at 13:00 local time. In certain cases where arrival is earlier or later, it may be necessary for the adaptation schedule to be advanced or delayed by a day, respectively. For example, for a 21:00 arrival after a $9 \mathrm{~h}$ westward time zone change, the day 0 schedule should be delayed to occur on day 1 because arrival would occur after the critical time for light exposure, so adaptation has to begin one day "late" (Figure 4C). Conversely, for a 06:00 arrival after a $6 \mathrm{~h}$ eastward time zone change, the day 1 schedule should be advanced to occur on day 0 because arrival would occur prior to the critical time for light exposure, so adaptation can begin one day "early" (Figure 5B).

When a schedule requires light exposure during the daytime, it is best to be outside in sunlight without sunglasses. When a schedule requires light exposure after sunset, bright indoor light, or a light box, or light-emitting glasses should be used. When a schedule requires light avoidance, it is best to be inside with lights off or as dim as possible-it may even be appropriate to have a nap (limited to $1 \mathrm{~h}$ so as not to interfere with night-time sleep). When a schedule requires light avoidance during the daytime and being outside is unavoidable, wrap-around sunglasses with minimal light transmission should be worn.

Complete adaptation after transmeridian flight is achieved when the circadian system shifts sufficiently such that CBTmin in the arrival time zone occurs at the same time as it occurred in the departure time zone (i.e., 04:00, assuming habitual bed time of 23:00-07:00). However, this can take several days, so from a practical point of view, it is most important that the circadian system shifts sufficiently such that CBTmin occurs during the main night-time sleep period in the new time zonea state of partial adaptation (Eastman and Burgess, 2009). Once CBTmin - the daily low-point of the circadian cycleoccurs during the night-time, sleep should be longer and of better quality, daytime sleepiness should be reduced, and mental and physical performance should be higher, i.e., the symptoms of jet lag should be greatly reduced. This distinction between complete and partial adaptation has previously been applied to shift workers switching from day work to night work (Lee et al., 2006).

NB. If melatonin is used by an athlete, a responsible party must ensure that it is not a prohibited substance under the relevant drug code and that it is sourced from a reputable supplier (to ensure purity and dose accuracy).

Adaptation schedules after westward flight:

- Time zone change of $3 \mathrm{~h}$ west (e.g., from Wellington, New Zealand, to Tokyo, Japan). Immediately after flight, CBTmin will occur at 01:00 local time, instead of 04:00, and CBTmax will occur at 13:00 local time, instead of 16:00. To adapt to the new time zone, the circadian system has to delay by $3 \mathrm{~h}-$ partial and complete adaptation should be achieved on days 1 and 4, respectively (Figure $\mathbf{4 A}$ ).

- Time zone change of $6 \mathrm{~h}$ west (e.g., from Anchorage, USA, to Tokyo, Japan). Immediately after flight, CBTmin will occur at 22:00 local time, instead of 04:00, and CBTmax will occur at 10:00 local time, instead of 16:00. To adapt to the new time zone, the circadian system has to delay by $6 \mathrm{~h}-$ partial and complete adaptation should be achieved on days 2 and 6 , respectively (Figure 4B).

- Time zone change of $9 \mathrm{~h}$ west (e.g., from Minneapolis, USA, to Tokyo, Japan). Immediately after flight, CBTmin will occur at 19:00 local time, instead of 04:00, and CBTmax will occur at 07:00 local time, instead of 16:00. To adapt to the new time zone, the circadian system has to delay by $9 \mathrm{~h}-$ partial and complete adaptation should be achieved on days 3 and 7 , respectively (Figure 4C).

Adaptation schedules after eastward flight:

- Time zone change of $3 \mathrm{~h}$ east (e.g., from Dhaka, Bangladesh, to Tokyo, Japan). Immediately after flight, CBTmin will occur at 07:00 local time, instead of 04:00, and CBTmax will occur at 19:00 local time, instead of 16:00. To adapt to the new time zone, the circadian system has to advance by $3 \mathrm{~h}-$ partial and complete adaptation should be achieved on days 1 and 4 , respectively (Figure 5A).

- Time zone change of $6 \mathrm{~h}$ east (e.g., from Doha, Qatar, to Tokyo, Japan). Immediately after flight, CBTmin will occur at 10:00 local time, instead of 04:00, and CBTmax will occur at 22:00 local time, instead of 16:00. To adapt to the new time zone, the circadian system has to advance by $6 \mathrm{~h}-$ partial and complete adaptation should be achieved on days 3 and 6 , respectively (Figure 5B).

- Time zone change of $9 \mathrm{~h}$ east (e.g., from London, United Kingdom, to Tokyo, Japan). Immediately after flight, CBTmin will occur at 13:00 local time, instead of 04:00, and CBTmax will occur at 01:00 local time, instead of 16:00. To adapt to the new time zone, the circadian system has to advance by $9 \mathrm{~h}$-partial and complete adaptation should be achieved on days 5 and 8, respectively (Figure 5C).

Adaptation schedules after a time zone change of $12 \mathrm{~h}$ east/west (e.g., from Buenos Aires, Argentina, to Tokyo, Japan):

- Immediately after flight, CBTmin will occur at 16:00 local time, instead of 04:00, and CBTmax will occur at 04:00 local time, instead of 16:00. To adapt to the new time zone, the circadian system could either delay or advance by $12 \mathrm{~h}$. However, given that the human circadian system has a natural period of $\sim 24.2 \mathrm{~h}$ such that it has a greater propensity to delay than to 


\section{A Time Zone Shift of $3 \mathrm{~h}$ West}

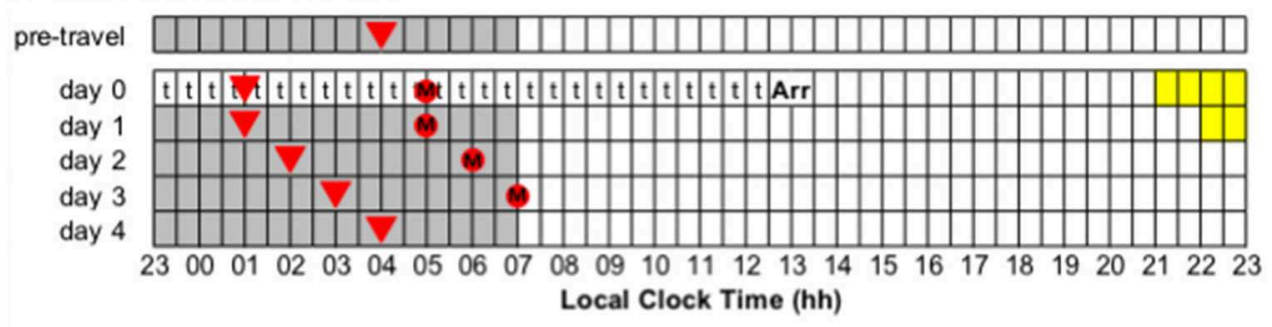

\section{B Time Zone Shift of $6 \mathrm{~h}$ West}

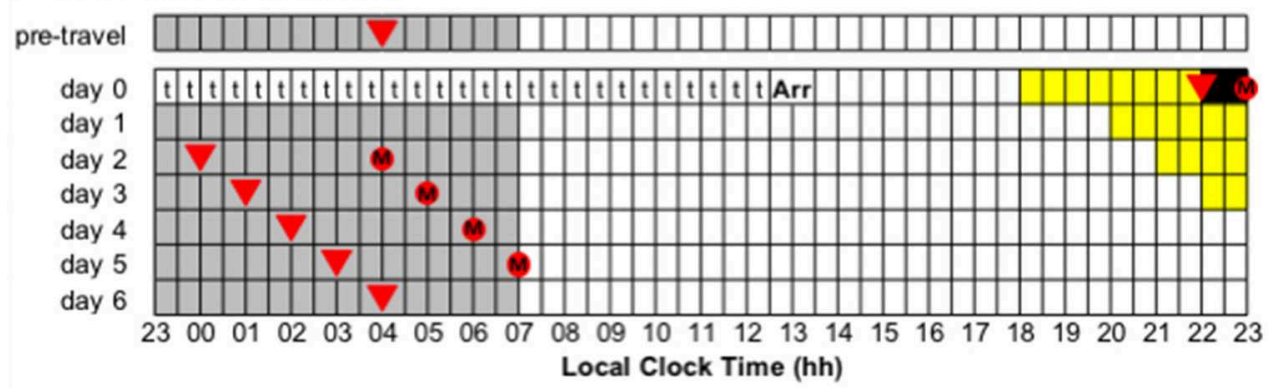

\section{Time Zone Shift of $9 \mathrm{~h}$ West}

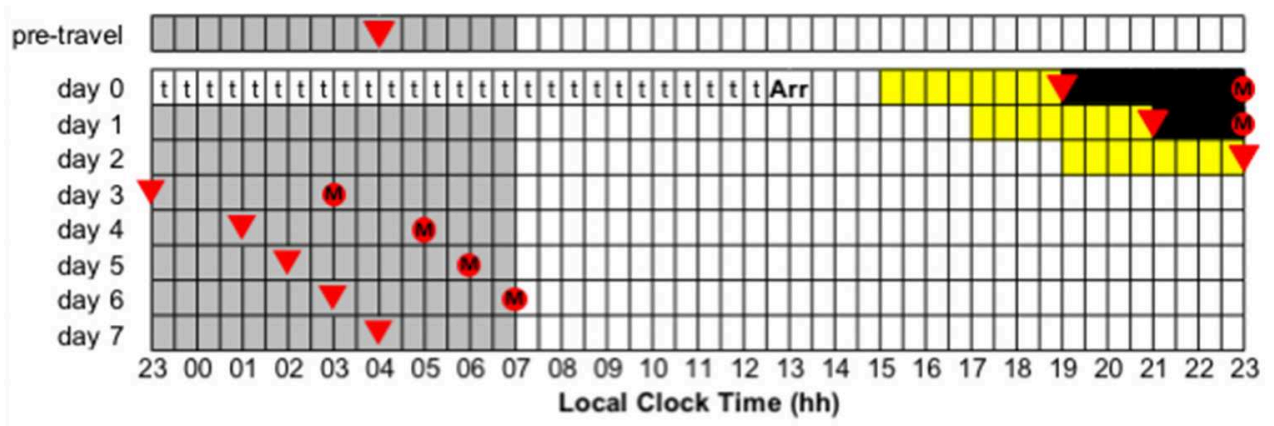

\section{Time Zone Shift of $12 \mathrm{~h}$ West}

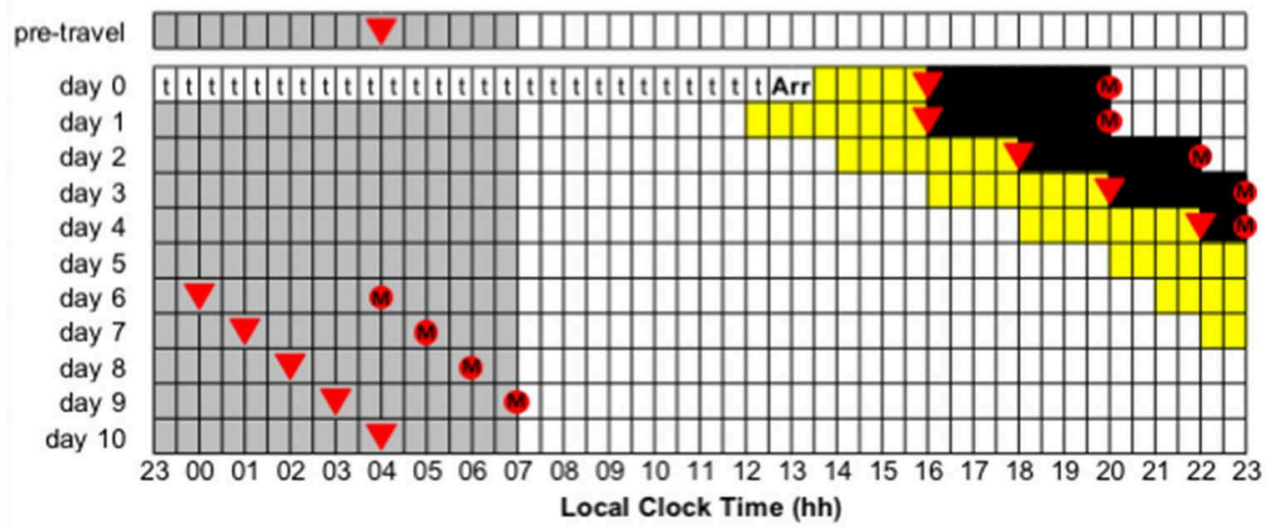

FIGURE 4 | Use of light exposure/avoidance and ingestion of exogenous melatonin to facilitate adaptation to westward time zone shifts of 3, 6, 9, and 12 h. In all panels, "t" represents transmeridian travel; "Arr" represents arrival in the new time zone; gray boxes represent sleep; white boxes represent wake; yellow boxes represent light exposure during wake; black boxes represent light avoidance during wake; red circles with an "M" represent ingestion of a 3.0 mg dose of exogenous melatonin; inverted triangles represent the daily minimum of core body temperature (CBTmin). Partial adaptation is achieved when CBTmin in the new time zone occurs within the scheduled sleep period, and complete adaptation is achieved when CBTmin in the new time zone occurs at the same time as the pre-travel CBTmin. On days when the ideal time of melatonin ingestion occurs during a scheduled sleep period, it should occur at that time if awake, otherwise it should be missed. This figure was inspired by similar figures presented by Eastman and Burgess (2009) and Revell and Eastman (2012). (A) Time zone shift of 3 h west. (B) Time zone shift of $6 \mathrm{~h}$ west. (C) Time zone shift of $9 \mathrm{~h}$ west. (D) Time zone shift of $12 \mathrm{~h}$ west. 


\section{A Time Zone Shift of $3 \mathrm{~h}$ East}

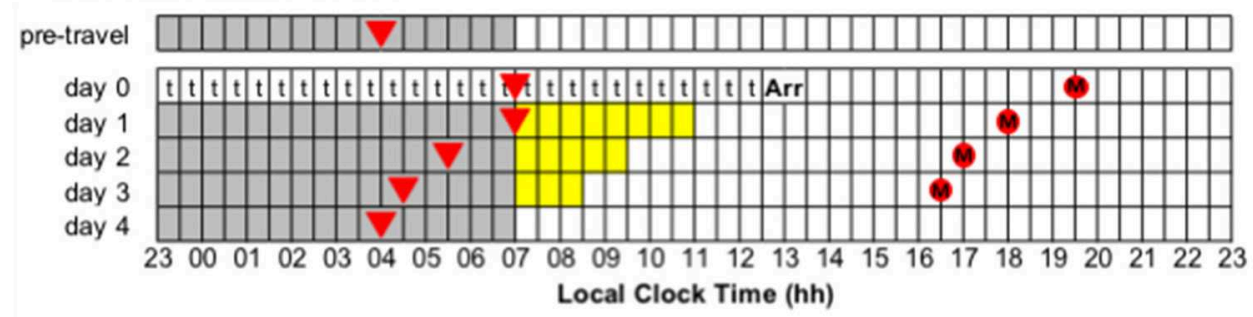

\section{B Time Zone Shift of $6 \mathrm{~h}$ East}

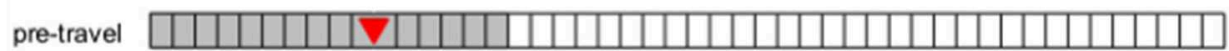

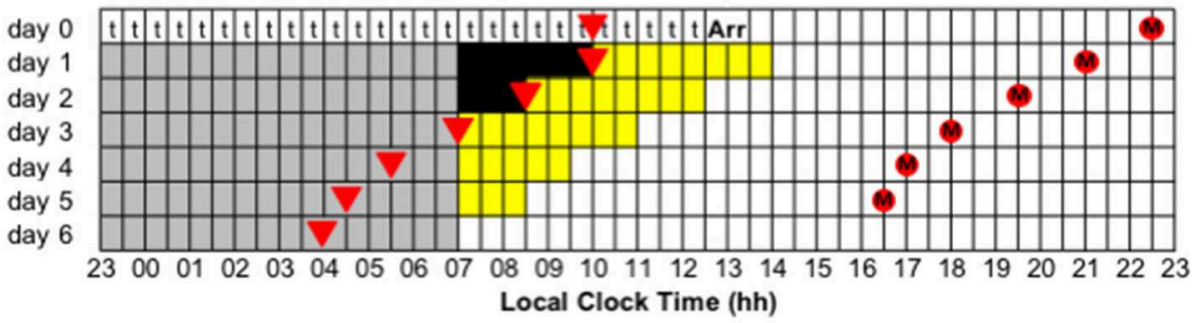

\section{Time Zone Shift of $9 \mathrm{~h}$ East}

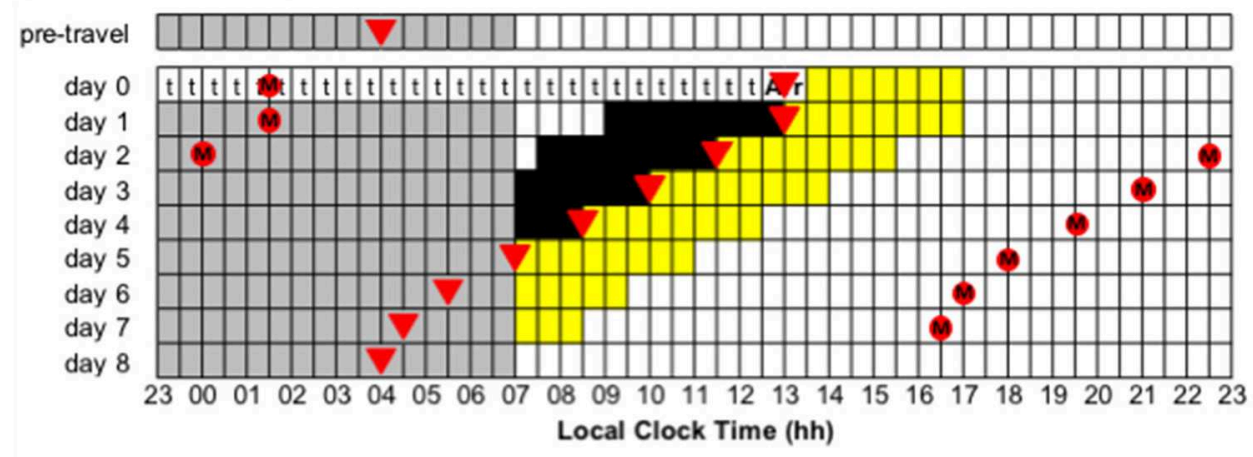

\section{Time Zone Shift of $12 \mathrm{~h}$ East}

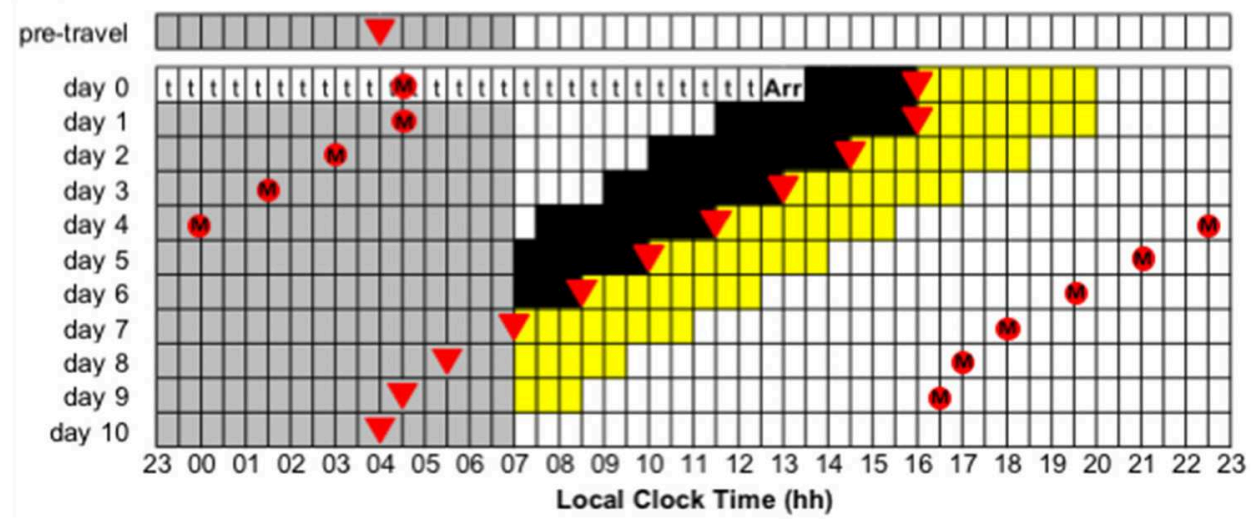

FIGURE 5 | Use of light exposure/avoidance and ingestion of exogenous melatonin to facilitate adaptation to eastward time zone shifts of 3, 6 , 9 , and $12 \mathrm{~h}$. In all panels, "t" represents transmeridian travel; "Arr" represents arrival in the new time zone; gray boxes represent sleep; white boxes represent wake; yellow boxes represent light exposure during wake; black boxes represent light avoidance during wake; red circles with an "M" represent ingestion of a 3.0 mg dose of exogenous melatonin; inverted triangles represent the daily minimum of core body temperature (CBTmin). Partial adaptation is achieved when CBTmin in the new time zone occurs within the scheduled sleep period, and complete adaptation is achieved when CBTmin in the new time zone occurs at the same time as the pre-travel CBTmin. On days when the ideal time of melatonin ingestion occurs during a scheduled sleep period, it should occur at that time if awake, otherwise it should be missed. This figure was inspired by similar figures presented by Eastman and Burgess (2009) and Revell and Eastman (2012). (A) Time zone shift of 3 h east. (B) Time zone shift of $6 \mathrm{~h}$ east. (C) Time zone shift of $9 \mathrm{~h}$ east. (D) Time zone shift of $12 \mathrm{~h}$ east. 
advance (Czeisler et al., 1999; Zhou et al., 2011b), it is more common to adapt by delay. Using a delay schedule, partial and complete adaptation should be achieved on days 6 and 10, respectively (Figure 4D). Using an advance schedule, partial and complete adaptation should be achieved on days 7 and 10, respectively (Figure 5D).

\section{EVIDENCE OF EFFICACY FOR JET LAG INTERVENTIONS BASED ON LIGHT AND/OR MELATONIN}

This manuscript contains recommendations regarding the use of judiciously timed light exposure/avoidance and ingestion of exogenous melatonin to minimize jet lag by facilitating adaptation of the circadian system to a new time zone. These recommendations are primarily based on information contained in phase response curves, which describe the effects of light and melatonin on the timing of the circadian system (Czeisler et al., 1989; Khalsa et al., 2003; Burgess et al., 2008, 2010). Computer-based simulations with experimentallyvalidated mathematical models have demonstrated that schedules of light exposure and avoidance could be used to increase the rate of adaptation after a rapid shift in the timing of the light-dark cycle (Serkh and Forger, 2014). Laboratory-based trials have established that both light and melatonin, when administered alone, can shift the timing of the circadian system (Deacon and Arendt, 1995; Middleton et al., 1997; Burgess et al., 2003a; Smith and Eastman, 2009), and when used in combination, the phase-shifting effects of light and melatonin are additive (Revell et al., 2006; Paul et al., 2011). Only a few field studies have been conducted to examine the efficacy of light-based interventions for the treatment of jet lag-and their results are equivocal (Boulos et al., 2002; Lahti et al., 2007; Thompson et al., 2012). In contrast, a meta-analysis of 10 field studies examining the efficacy of melatonin-based interventions indicates that they are effective at shifting the body clock and at reducing subjective ratings of jet lag (Herxheimer and Petrie, 2002). To date, the efficacy of combined light/melatonin interventions has not been assessed in field-based settings, so this is a critical next step for the advancement of knowledge in this field.

\section{COULD ADAPTATION BEGIN BEFORE/DURING TRANSMERIDIAN TRAVEL?}

Specific guides have not been provided here, but it is possible to begin shifting the circadian system in the desired direction before and/or during transmeridian flight (see Eastman and Burgess, 2009; Revell and Eastman, 2012). The potential advantage of preshifting is that it should reduce the amount of time required to adapt to local time cues in the arrival time zone, such that the symptoms of jet lag are less pronounced and/or occur over fewer days. Conversely, the potential disadvantages of pre-shifting are that it could interfere with sleep and be socially disruptive prior to travel and it could make it more difficult to estimate the timing of the daily minimum in core body temperature (CBTmin), and thus the appropriate times for light exposure/avoidance and/or melatonin ingestion, upon arrival in the new time zone.

To delay the circadian system in the 3-4 days prior to westward travel, gradually move bedtime and get-up time later (i.e., 30-60 min per day), maximize evening light exposure, minimize morning light exposure, and take $3.0 \mathrm{mg}$ of melatonin $1 \mathrm{~h}$ after rising from bed. To advance the circadian system in the 3-4 days prior to eastward travel, gradually move bedtime and get-up time earlier (i.e., 30-60 min per day), minimize evening light exposure, maximize morning light exposure, and take $3.0 \mathrm{mg}$ of melatonin $6.5 \mathrm{~h}$ before bed.

To shift the circadian system during travel, rather than setting a watch and attempting to align sleep and wake with the arrival time zone, light exposure/avoidance and melatonin ingestion should be timed according to the departure time zone. To delay the circadian system during westward travel, maximize light exposure in the $\sim 3 \mathrm{~h}$ before CBTmin, avoid light in the $\sim 3 \mathrm{~h}$ after CBTmin (by sleeping if possible), and take $3.0 \mathrm{mg}$ of melatonin $4 \mathrm{~h}$ after CBTmin. To advance the circadian system during eastward travel, take $3.0 \mathrm{mg}$ of melatonin $11.5 \mathrm{~h}$ before CBTmin, avoid light in the $\sim 3 \mathrm{~h}$ prior to CBTmin (by sleeping if possible), and maximize light exposure in the $\sim 3 \mathrm{~h}$ after CBTmin.

\section{CONCLUSIONS}

Long-haul flight over several time zones causes both travel fatigue and jet lag. The most obvious consequences of jet lag are poor sleep at night, excessive sleepiness during the day, and poor mental and physical performance. These consequences occur because the human circadian system cannot immediately adapt to time cues in a new time zone. This manuscript has presented recommendations on how to minimize jet lag using judiciously timed light exposure/avoidance and ingestion of exogenous melatonin to facilitate adaptation of the circadian system to a new time zone. These recommendations are based on the latest information regarding the effects of light and melatonin on the human circadian system. There are potential barriers to the practical implementation of these recommendations, so it will be critical to assess their efficacy in natural settings, preferably using experimental designs with randomization to treatment and control groups.

\section{AUTHOR CONTRIBUTIONS}

GR and CS contributed to conception of the manuscript. GR wrote the first draft of the manuscript. GR and CS contributed to revision of the manuscript. GR and CS read and approved the submitted version of the manuscript.

\section{FUNDING}

The authors are grateful for funding to support this manuscript from the Australian Research Council's Discovery Project scheme (DP160104909). 


\section{REFERENCES}

Arendt, J., and Skene, D. J. (2005). Melatonin as a chronobiotic. Sleep Med. Rev. 9, 25-39. doi: 10.1016/j.smrv.2004.05.002

Aschoff, J., Fatranska, M., Giedke, H., Doerr, P., Stamm, D., and Wisser, H. (1971). Human circadian rhythms in continuous darkness: entrainment by social cues. Science 171, 213-215. doi: 10.1126/science.171.3967.213

Bass, J. (2012). Circadian topology of metabolism. Nature 491, 348-356. doi: 10.1038 /nature1 1704

Boivin, D. B., Duffy, J. F., Kronauer, R. E., and Czeisler, C. A. (1996). Doseresponse relationships for resetting of human circadian clock by light. Nature 379, 540-542. doi: 10.1038/379540a0

Boulos, Z., Macchi, M. M., Stürchler, M. P., Stewart, K. T., Brainard, G. C., Suhner, A., et al. (2002). Light visor treatment for jet lag after westward travel across six time zones. Aviat. Space Envir. Med. 73, 953-963.

Brown, E. N., Choe, Y., Shanahan, T. L., and Czeisler, C. A. (1997). A mathematical model of diurnal variations in human plasma melatonin levels. Am. J. Physiol. 272, E506-E516. doi: 10.1152/ajpendo.1997.272.3.e506

Brown, T. P., Shuker, L. K., Rushton, L., Warren, F., and Stevens, J. (2001). The possible effects on health, comfort and safety of aircraft cabin environments. J. R. Soc. Promo. Health 121, 177-184. doi: 10.1177/1466424001121 00315

Burgess, H. J., Crowley, S. J., Gazda, C. J., Fogg, L. F., and Eastman, C. I. (2003a). Preflight adjustment to eastward travel: 3 days of advancing sleep with and without morning bright light. J. Biol. Rhythms 18, 318-328. doi: $10.1177 / 0748730403253585$

Burgess, H. J., and Eastman, C. E. (2005). The dim light melatonin onset following fixed and free sleep schedules. J. Sleep. Res. 14, 229-237. doi: $10.1111 / j .1365-2869.2005 .00470 . x$

Burgess, H. J., Revell, V. L., and Eastman, C. I. (2008). A three pulse phase response curve to three milligrams of melatonin in humans. J. Physiol. 586, 639-647. doi: 10.1113/jphysiol.2007.143180

Burgess, H. J., Revell, V. L., Molina, T. A., and Eastman, C. I. (2010). Human phase response curves to three days of daily melatonin: $0.5 \mathrm{mg}$ versus $3.0 \mathrm{mg}$. Endocr. Res. 95, 3325-3331. doi: 10.1210/jc.2009-2590

Burgess, H. J., Savic, N., Sletten, T., Roach, G. D., Gilbert, S. S., and Dawson, D. (2003b). The relationship between the dim light melatonin onset and sleep on a regular schedule in young healthy adults. Behav. Sleep Med. 1, 102-114. doi: $10.1207 /$ s15402010bsm0102_3

Burke, T. M., Markwald, R. R., Chinoy, E. D., Snider, J. A., Bessman, S. C., Jung, C. M., et al. (2013). Combination of light and melatonin time cues for phase advancing the human circadian clock. Sleep 36, 1617-1624. doi: $10.5665 /$ sleep. 3110

Buxton, O. M., Lee, C. W., L'Hermite-Baleriaux, M., Turek, F. W., and Van Cauter, E. (2003). Exercise elicits phase shifts and acute alterations of melatonin that vary with circadian phase. Am. J. Physiol. Reg. I. 284, R714-R724. doi: 10.1152/ajpregu.00355.2002

Cagnacci, A., Soldani, R., Laughlin, G. A., and Yen, S. S. C. (1996). Modification of circadian body temperature rhythm during the luteal menstrual phase: role of melatonin. J. Appl. Physiol. 80, 25-29. doi: 10.1152/jappl.1996.80.1.25

Czeisler, C. A., Allan, J. S., Strogatz, S. H., Ronda, J. M., Sanchez, R., Rios, C. D., et al. (1986). Bright light resets the human circadian pacemaker independent of the timing of the sleep-wake cycle. Science 233, 667-671. doi: $10.1126 /$ science. 3726555

Czeisler, C. A., Duffy, J. F., Shanahan, T. L., Brown, E. N., Mitchell, J. F., Rimmer, D. W., et al. (1999). Stability, precision, and near-24hour period of the human circadian pacemaker. Science 284, 2177-2181. doi: $10.1126 /$ science. 284.5423 .2177

Czeisler, C. A., Kronauer, R. E., Allan, J. S., Duffy, J. F., Jewett, M. E., Brown, E. N., et al. (1989). Bright light induction of strong (type 0) resetting of the human circadian pacemaker. Science 244, 1328-1333. doi: 10.1126/science.2734611

Darwent, D., Ferguson, S. A., Sargent, C., Paech, G. M., Williams, L., Zhou, X., et al. (2010). Contribution of core body temperature, prior wake time, and sleep stages to cognitive throughput performance during forced desynchrony. Chronobiol. Int. 27, 898-910. doi: 10.3109/07420528.2010.488621

Deacon, S., and Arendt, J. (1995). Melatonin-induced temperature suppression and its acute phase-shifting effects correlate in a dose-dependent manner in humans. Brain Res. 688, 77-85. doi: 10.1016/0006-8993(95)96872-i
Dijk, D.-J., and Czeisler, C. A. (1995). Contribution of the circadian pacemaker and the sleep homeostat to sleep propensity, sleep structure, electroencephalographic slow waves, and sleep spindle activity in humans. J. Neurosci. 15, 3526-3538. doi: 10.1523/jneurosci.15-05-03526.1995

Dijk, D.-J., Duffy, J. F., and Czeisler, C. A. (1992). Circadian and sleep/wake dependent aspects of subjective alertness and cognitive performance. J. Sleep Res. 1, 112-117. doi: 10.1111/j.1365-2869.1992.tb00021.x

Eastman, C. I., and Burgess, H. J. (2009). How to travel the world without jet lag. Sleep Med. Clin. 4, 241-255. doi: 10.1016/j.jsmc.2009.02.006

Eastman, C. I., Hoese, E. K., Youngstedt, S. D., and Liu, L. (1995). Phase-shifting human circadian rhythms with exercise during the night shift. Physiol. Behav. 58, 1287-1291. doi: 10.1016/0031-9384(95)02031-4

Eastman, C. I., Martin, S. K., and Hebert, M. (2000). Failure of extraocular light to facilitate circadian rhythm reentrainment in humans. Chronobiol. Int. 17, 807-826. doi: $10.1081 /$ cbi-100102116

Ferguson, S. A., Paech, G. M., Sargent, C., Darwent, D., Kennaway, D. J., and Roach, G. D. (2012). The influence of circadian time and sleep dose on subjective fatigue ratings. Accident Anal. Prev. 45, 50-54. doi: 10.1016/j.aap.2011.09.026

Halberg, F. (1959). Physiologic 24-hour periodicity: general and procedural considerations with reference to the adrenal cycle. Z. Vit. Hormone Ferment. $10,225-296$.

Herxheimer, A., and Petrie, K. J. (2002). Melatonin for the prevention and treatment of jet lag. Cochrane Db. Syst. Rev. doi: 10.1002/14651858.cd001520

Khalsa, S.-B. S., Jewett, M. E., Cajochen, C., and Czeisler, C. A. (2003). A phase response curve to single bright light pulses in human subjects. J. Physiol. 549, 945-952. doi: 10.1113/jphysiol.2003.040477

Kosmadopoulos, A., Sargent, C., Darwent, D., Zhou, X., Dawson, D., and Roach, G. D. (2014). The effects of a split sleep-wake schedule on neurobehavioural performance and predictions of performance under conditions of forced desynchrony. Chronobiol. Int. 31, 1209-1217. doi: 10.3109/07420528.2014.957763

Kosmadopoulos, A., Sargent, C., Zhou, X., Darwent, D., Matthews, R. W., Dawson, D., et al. (2017). The efficacy of objective and subjective predictors of driving performance during sleep restriction and circadian misalignment. Accident Anal. Prev. 99, 445-451. doi: 10.1016/j.aap.2015. 10.014

Lahti, T., Terttunen, J., Leppämäki, S., Lönnqvist, J., and Partonen, T. (2007). Field trial of timed bright light exposure for jet lag among airline cabin crew. Int. J. Circumpolar Health 66, 365-369. doi: 10.3402/ijch.v66i4. 18280

Lavie, P. (1986). Ultradian rhythms in human sleep. III. 'Gates' and 'Forbidden zones' for sleep. Electroen. Clin. Neuro. 63, 414-425. doi: 10.1016/0013-4694(86)90123-9

Lee, C., Smith, M. R., and Eastman, C. I. (2006). A compromise phase position for permanent night shift workers: circadian phase after two night shifts with scheduled sleep and light/dark exposure. Chronobiol. Int. 23, 859-875. doi: $10.1080 / 07420520600827160$

Matthews, R. W., Ferguson, S. A., Zhou, X., Darwent, D., Sargent, C., Kennaway, D. J., et al. (2010). The interactive influence of the internal body clock and prior wake on task vigilance. Ergonomia 32, 37-48.

Matthews, R. W., Ferguson, S. A., Zhou, X., Kosmadopoulos, A., Kennaway, D. J., and Roach, G. D. (2012a). Simulated driving under the influence of extended wake, time of day and sleep restriction. Accident Anal. Prev. 45, 55-61. doi: 10.1016/j.aap.2011.09.027

Matthews, R. W., Ferguson, S. A., Zhou, X., Sargent, C., Darwent, D., Kennaway, D. J., et al. (2012b). Time-of-day mediates the influences of extended wake and sleep restriction on simulated driving. Chronobiol. Int. 29, 572-579. doi: 10.3109/07420528.2012.675845

Middleton, B., Arendt, J., and Stone, B. (1997). Complex effects of melatonin on human circadian rhythms in constant dim light. J. Biol. Rhythms 12, 467-475. doi: $10.1177 / 074873049701200508$

Mistlberger, R. E., and Skene, D. J. (2004). Social influences on mammalian circadian rhythms: animal and human studies. Biol. Rev. 79, 533-556. doi: $10.1017 / \mathrm{s} 1464793103006353$

Paech, G. M., Ferguson, S. A., Sargent, C., Darwent, D., Williams, L., Kennaway, D. J., et al. (2010). A 28-hour day, sleep, and a single beat period: revisiting forced desynchrony studies? Ergonomia 32, 125-132. 
Paech, G. M., Ferguson, S. A., Sargent, C., Kennaway, D. J., and Roach, G. D. (2012). The relative contributions of the homeostatic and circadian processes to sleep regulation under conditions of severe sleep restriction. Sleep 35, 941-948. doi: 10.5665/sleep.1956

Paul, M. A., Gray, G. W., Lieberman, H. R., Love, R. J., Miller, J. C., Trouborst, M., et al. (2011). Phase advance with separate and combined melatonin and light treatment. Psychopharmacology 214, 515-523. doi: 10.1007/s00213-010-2059-5

Reilly, T., Atkinson, G., and Waterhouse, J. (1997). Biological Rhythms and Exercise. Oxford: Oxford University Press.

Revell, V. L., Burgess, H. J., Gazda, C. J., Smith, M. R., Fogg, L. F., and Eastman, C. I. (2006). Advancing human circadian rhythms with afternoon melatonin and morning intermittent bright light. J. Clin. Endocr. Metab. 91, 54-59. doi: 10.1210/jc.2005-1009.

Revell, V. L., and Eastman, C. I. (2012). "Jet lag and its prevention," in Therapy in Sleep Medicine, eds T. J. Barkoukis, J. K. Matheson, R. Ferber, and K. Doghramji (Philadelphia, PA: Elsevier), 390-401.

Rimmer, D. W., Boivin, D. B., Shanahan, T. L., Kronauer, R. E., Duffy, J. F., and Czeisler, C. A. (2000). Dynamic resetting of the human circadian pacemaker by intermittent bright light. Am. J. Physiol. Reg. I. 279, R1574R1579. doi: 10.1152/ajpregu.2000.279.5.r1574

Roach, G. D., Matthews, R., Naweed, A., Kontou, T. G., and Sargent, C. (2018). Flat-out napping: the quantity and quality of sleep obtained in a seat during the daytime increase as the angle of recline of the seat increases. Chronobiol. Int. 35, 872-883. doi: 10.1080/07420528.2018.1466801

Rüger, M., St. Hilaire, M. A., Brainard, G. C., Khalsa, S.-B. S., Kronauer, R. E., Czeisler, C. A., et al. (2013). Human phase response curve to single $6.5 \mathrm{~h}$ pulse short-wavelength light. J. Physiol. 59, 353-363. doi: 10.1113/jphysiol.2012.239046

Sargent, C., Darwent, D., Ferguson, K. D. J., and Roach, G. D. (2012a). Sleep restriction masks the influence of the circadian process on sleep propensity. Chronobiol. Int. 29, 565-571. doi: 10.3109/07420528.2012.675256

Sargent, C., Darwent, D., Ferguson, S. A., and Roach, G. D. (2012b). Can a simple balance task be used to assess fitness for duty? Accident Anal. Prev. 45, 74-79. doi: 10.1016/j.aap.2011.09.030

Sargent, C., Ferguson, S. A., Darwent, D., Kennaway, D. J., and Roach, G. D. (2010). The influence of circadian phase and prior wake on neuromuscular function. Chronobiol. Int. 27, 911-921. doi: 10.3109/07420528.2010.488901

Sargent, C., Zhou, X., Matthews, R. W., Darwent, D., and Roach, G. D. (2016). Daily rhythms of hunger and satiety in healthy men during one week of sleep restriction and circadian misalignment. Int. J. Env. Res. 13:e170. doi: 10.3390/ijerph13020170

Scheer, F. A. J. L., Hilton, M. F., Mantzoros, C. S., and Shea, S. A. (2009). Adverse metabolic and cardiovascular consequences of circadian misalignment. Proc. Natl. Acad. Sci. U.S.A. 106, 4453-4458. doi: 10.1073/pnas.0808180106

Scheer, F. A. J. L., Hu, K., Evoniuk, H., Kelly, E. E., Malhotra, A., Hilton, M. F., et al. (2010). Impact of the human circadian system, exercise, and their interaction on cardiovascular function. Proc. Natl. Acad. Sci. U.S.A. 107, 20541-20546. doi: 10.1073/pnas.1006749107

Schibler, U., Gotic, I., Saini, C., Gos, P., Curie, T., Emmenegger, Y., et al. (2015). Clock-talk: interactions between central and peripheral circadian oscillators in mammals. Cold SH. Q. B. 80, 223-232. doi: 10.1101/sqb.2015.80.027490

Serkh, K., and Forger, D. B. (2014). Optimal schedules of light exposure for rapidly correcting circadian misalignment. PLoS Comput. Biol. 10:e1003523. doi: 10.1371/journal.pcbi.1003523

Smith, M. R., and Eastman, C. I. (2009). Phase delaying the human circadian clock with blue-enriched polychromatic light. Chronobiol. Int. 26, 709-725. doi: 10.1080/07420520902927742

Thompson, A., Batterham, A., Jones, H., Gregson, W., Scott, D., and Atkinson, G. (2012). The practicality and effectiveness of supplementary bright light for reducing jet-lag in elite female athletes. Int. J. Sports Med. 34, 582-589. doi: $10.1055 / \mathrm{s}-0032-1331160$

Ueda, H. R., Chen, W., Minami, Y., Honma, S., Honma, K., Iino, M., et al. (2004). Molecular-timetable methods for detection of body time and rhythm disorders from single-time-point genome-wide expression profiles. Proc. Natl. Acad. Sci. U.S.A. 101, 11227-11232. doi: 10.1073/pnas.0401882101 van den Heuvel, C. J., Ferguson, S. A., Macchi, M. M., and Dawson, D. (2005). Melatonin as a hypnotic: con. Sleep Med. Rev. 9, 71-80. doi: 10.1016/j.smrv.2004.07.001

Waterhouse, J., Reilly, T., Atkinson, G., and Edwards, B. (2007). Jet lag: trends and coping strategies. Lancet 369, 1117-1129. doi: 10.1016/s0140-6736(07)60529-7

Waterhouse, J., Reilly, T., and Edwards, B. (2004). The stress of travel. J. Sport. Sci. 22, 946-966. doi: 10.1080/02640410400000264

Wever, R. A. (1980). Phase shifts of human circadian rhythms due to shifts of artificial zeitgebers. Chronobiologia 7, 303-327.

Wever, R. A., Polasek, J., and Wildgruber, C. M. (1983). Bright light affects human circadian rhythms. Pflug. Arch. - Eur. J. Phy. 396, 85-87. doi: 10.1007/bf00584704

Winget, C. M., DeRoshia, C. W., Markley, C. L., and Holley, D. C. (1984). A review of human physiological and performance changes associated with desynchronosis of biological rhythms. Aviat. Space Envir. Med. 55, 1085-1096.

Wirz-Justice, A., Krauchi, K., Cajochen, C., Danilenko, K. V., Renz, C., and Weber, J. M. (2004). Evening melatonin and bright light administration induce additive phase shifts in dim light melatonin onset. J. Pineal Res. 36, 192-194. doi: 10.1111/j.1600-079x.2004.00117.x

Wittenbrink, N., Ananthasubramaniam, B., Münch, M., Koller, B., Maier, B., Weschke, C. et al. (2018). High-accuracy determination of internal circadian time from a single blood sample. J. Clin. Invest. 128, 3826-3839. doi: 10.1172/JCI120874

Wright, H. R., and Lack, L. C. (2001). Effect of light wavelength on suppression and phase delay of the melatonin rhythm. Chronobiol. Int. 18, 801-808. doi: $10.1081 / \mathrm{cbi}-100107515$

Wyatt, J. K., Dijk, D.-J., Ritz-De Cecco, A., Ronda, J. M., and Czeisler, C. A. (2006). Sleep-facilitating effect of exogenous melatonin in healthy young men and women is circadian-phase dependent. Sleep 29, 609-618. doi: 10.1093/sleep/29.5.609

Yamada, Y., and Forger, D. (2010). Multiscale complexity in the mammalian circadian clock. Curr. Opin. Genet. Dev. 20, 626-633. doi: 10.1016/j.gde.2010.09.006

Youngstedt, S. D., Elliott, J. A., and Kripke, D. F. (2019). Human circadian phaseresponse curves for exercise. J. Physiol. 597, 2253-2268. doi: 10.1113/jp276943

Zhdanova, I. V. (2005). Melatonin as a hypnotic: pro. Sleep Med. Rev. 9, 51-65. doi: 10.1016/j.smrv.2004.04.003

Zhou, X., Ferguson, S. A., Matthews, R. W., Sargent, C., Darwent, D., Kennaway, D. J., et al. (2011a). Dynamics of neurobehavioral performance variability under forced desynchrony: evidence of state instability. Sleep 34, 57-63. doi: 10.1093/sleep/34.1.57

Zhou, X., Ferguson, S. A., Matthews, R. W., Sargent, C., Darwent, D., Kennaway, D. J., et al. (2011b). Sleep, wake and phase dependent changes in neurobehavioral function under forced desynchrony. Sleep 34, 931-941. doi: 10.5665/sleep.1130

Zhou, X., Ferguson, S. A., Matthews, R. W., Sargent, C., Darwent, D., Kennaway, D. J., et al. (2012). Mismatch between subjective alertness and objective performance under sleep restriction is greatest during the biological night. J. Sleep Res. 21, 40-49. doi: 10.1111/j.1365-2869.2011. 00924.x

Zhou, X., Sargent, C., Kosmadopoulos, A., Darwent, D., Dawson, D., and Roach, G. D. (2017). Do split sleep/wake schedules reduce or increase sleepiness for continuous operations? Accident Anal. Prev. 99, 434-439. doi: 10.1016/j.aap.2015.10.027

Conflict of Interest Statement: The authors declare that the research was conducted in the absence of any commercial or financial relationships that could be construed as a potential conflict of interest.

Copyright (c) 2019 Roach and Sargent. This is an open-access article distributed under the terms of the Creative Commons Attribution License (CC BY). The use, distribution or reproduction in other forums is permitted, provided the original author(s) and the copyright owner(s) are credited and that the original publication in this journal is cited, in accordance with accepted academic practice. No use, distribution or reproduction is permitted which does not comply with these terms. 